Bull. Austral. Math. Soc.

VoL. 51 (1995) [83-86]

\title{
BISTABLE VECTOR FIELDS ARE AXIOM A
}

\author{
MIKE HURLEY
}

\begin{abstract}
Recently $\mathrm{L}$. Wen showed that if a $C^{1}$ vector field (on a smooth compact manifold without boundary) is both structurally stable and topologically stable then it will satisfy Axiom A. The purpose of this note is to indicate how results from an earlier paper can be used to simplify somewhat Wen's argument.
\end{abstract}

In [5] it was shown that if a $C^{1}$ diffeomorphism on a compact manifold is bistable (that is, both topologically stable and structurally stable), then the diffeomorphism is an Axiom A diffeomorphism. The proof goes by showing that such a diffeomorphism satisfies conditions that Mañé had shown [8] would imply Axiom A. (This result from [5] was later superseded by Mañé's solution of the $C^{1}$ stability conjecture that structural stability alone implied Axiom A for diffeomorphisms [9].) Later, Chi, Choi and Park [2] addressed the question of whether bistability implies Axiom $A$ for vector fields, and announced that it does (the stability conjecture for vector fields in dimensions greater than 3 is still open). There were some problems with their approach, as described by Wen in [12]. In addition to pointing out the difficulties with the argument in [2], Wen shows that bistability implies Axiom A for vector fields. The proof goes by checking that a bistable vector field satisfies conditions that Liao [7] has shown to imply Axiom A. For the most part, Wen's argument follows the same general lines as in the diffeomorphism case, using results from $[4,5]$; however it introduces a special argument (Lemma 4) to deal with the singular (fixed) points of the vector field. The point of this paper is to show that the special argument dealing with the fixed points can be replaced by a simpler argument from [6] that is more in the spirit of the rest of Wen's proof. (The results of [6] are given in terms of flows rather than vector fields, but this is not important as the same proofs work in either context.) For the most part we shall refer the reader to [12] for detailed definitions and background.

The theorem of Liao that is the key to establishing Axiom $A$ is given below. To say that a vector field is Axiom A means that its nonwandering set carries a hyperbolic structure and is the closure of its closed orbits (a closed orbit is either a fixed point or a nontrivial periodic orbit). See $[2,11,12]$ for a more detailed description of Axiom A. If $Z$ is a vector field, let $\Lambda_{i}(Z)$ denote the closure of the set of (non-fixed) hyperbolic

Received 21st March, 1994

Copyright Clearance Centre, Inc. Serial-fee code: 0004-9729/95 $\$$ \$2.00+0.00. 
periodic orbits of $Z$ whose unstable manifolds have dimension $i$, and let $\Sigma(Z)$ be the set of hyperbolic fixed points of $Z$.

Theorem 1. Suppose that $V$ is a $C^{1}$ vector field on the compact manifold $M$ that has a $C^{1}$ neighbourhood $\mathcal{W}$ with the following properties:

(1) If $Z \in \mathcal{W}$ then all closed orbits of $Z$ are hyperbolic.

(2) If $Z \in \mathcal{W}$ then the sets $\Lambda_{i}(Z)(1 \leqslant i \leqslant \operatorname{dim}(M))$ and $\Sigma(Z)$ are strongly disjoint, meaning that there are fixed, pairwise disjoint, open subsets $U_{i}$ of $M$ with the property that $\Sigma(Z) \subset U_{0}$ and $\Lambda_{i}(Z) \subset U_{i}$ for all $Z \in W$.

Then $V$ satisfies Axiom $A$.

The chain recurrent set of a vector field $Z$ will be denoted $R(Z)$; its definition can be found in $[3,4,6,12] . R(Z)$ is a compact, nonempty, flow-invariant subset of $M$ that contains all closed orbits. The connected components of $R(Z)$ are called chain components (a different but equivalent definition was used in $[2,12]$; a proof of the equivalence of the two definitions can be found in [6]). A vector field $V$ is structurally stable if every vector field $Z$ in some $C^{1}$ neighbourhood of $V$ generates a flow that is topologically equivalent to the flow generated by $V . V$ is topologically stable if every vector field $Z$ in some $C^{0}$ neighbourhood of $V$ generates a flow that is semiconjugate to the flow of $V$. See $[4,6,12]$ for more details.

Theorem 2. Suppose that $V$ is a topologically stable vector field that satisfies the Kupka-Smale condition (that is, all of its closed orbits are hyperbolic, and the stable manifold of any closed orbit is transverse to the unstable manifold of any other closed orbit).

(1) The closed orbits of $V$ are dense in its chain recurrent set.

(2) $V$ has only finitely many chain components.

(3) $V$ has a finite number of fixed points, each of which is a single chain component of $V$.

(4) If $X$ is a chain component of $V$ that is not a single closed orbit, then $X$ is "fully tangled"; that is, if $\gamma_{1}$ and $\gamma_{2}$ are distinct periodic orbits in $X$, then the stable manifold of $\gamma_{1}$ intersects the unstable manifold of $\gamma_{2}$.

(5) Each $\Lambda_{i}(V)$ is a union of chain components.

(6) The sets $\Lambda_{i}(V)$ are pairwise disjoint.

ProOF: Conclusions 1 and 2 are contained in Theorem $\mathrm{C}$ of [4]. Conclusions 3 , 4 and 5 are, respectively, Corollary 7, Proposition 4, and Proposition 5 of [6]. It now follows that each set $\Lambda_{i}(V)$ is either empty or is the union of some (finite) number of chain components. Since the chain components are by definition pairwise disjoint, conclusion 6 follows.

Note that because the Kupka-Smale condition is necessary for structural stability, 
any bistable vector field satisfies the hypotheses of the last theorem. (The fact that $C^{1}$ structural stability implies the Kupka-Smale condition is well-known; it is an exercise of $[11$, page 114], and is discussed in $[1,10]$.)

Theorem 3. Any $C^{1}$ bistable vector field $V$ satisfies Axiom $A$.

Proof: We show that $V$ satisfies the hypotheses of Liao's theorem. Liao's first hypothesis (that all fixed points and periodic orbits of all nearby vector fields are hyperbolic) follows immediately from the assumption that $V$ is structurally stable. This is because structural stability is an open condition $\left(C^{1}\right)$ and structural stability implies the Kupka-Smale condition. To finish we must verify Liao's second hypothesis, the strong separation of the sets $\Lambda_{i}(Z), \Sigma(Z)$. Theorem 2 shows that $V$ has only finitely many chain components $X_{j}$; these are compact and pairwise disjoint. It follows that there are open sets $N_{j}$ whose closures are pairwise disjoint and with $X_{j} \subset N_{j}$ for each $j$. To extend the separation for $V$ that is provided by Theorem 2 to the strong separation for all $Z$ near $V$, we use the following.

LEMMA 4. The map $Z \rightarrow R(Z)$ is upper semicontinuous as a map from the set of $C^{1}$ vector fields into the set of closed subsets of $M$ provided with the Hausdorff topology.

The lemma follows from Conley's characterisation of the chain recurrent set in terms of the attractors of $V$ and their basins of attraction. See 6.2.A on page 37 of [3] for a proof of the lemma. The content of the lemma is that if $U$ is a neighbourhood of $R(V)$ then $U$ will also contain $R(Z)$ for all $Z$ sufficiently close to $V$.

Using the lemma and the remarks that preceded it, we see that there is a $C^{1}$ neighbourhood $\mathcal{W}$ of $V$ with any $Z \in \mathcal{W}$ satisfying the following properties:

(i) $Z$ is topologically conjugate to $V$;

(ii) all of the closed orbits orbits of $Z$ are hyperbolic;

(iii) $R(Z) \subset N \equiv \bigcup N_{j}$;

(iv) for each $j$ there is a hyperbolic closed orbit $\gamma_{j}(Z) \subset N_{j}$ that has the same type (that is, fixed point or periodic orbit) and index (the dimension of the stable manifold) as $\gamma_{j}(V)$.

(Part (iv) follows from the fact that Theorem 2 shows that $V$ has a hyperbolic closed orbit in each of its chain components, combined with the local stability of hyperbolic closed orbits [11].)

The fact that $Z$ is conjugate to $V$ means that the two vector fields have the same number of chain components. It also means that each chain component of $Z$ is either a single closed orbit or else is fully tangled as in part 4 of Theorem 2 . Let $X_{j}(Z)$ denote the chain component of $Z$ that contains $\gamma_{j}(Z)$, so $\gamma_{j}(Z) \subset X_{j}(Z) \subset N_{j}$. The transversality part of the Kupka-Smale condition can now be used to show that all 
periodic orbits in $X_{j}(Z)$ have the same index; see $[5,6]$ or $[12]$ for the details of the argument. This establishes the second of Liao's conditions, and so shows that a bistable vector field satisfies Axiom A.

\section{REFERENCES}

[1] R. Abraham and J.E. Marsden, Foundations of mechanics (Benjamin-Cummings, Reading MA, 1978).

[2] D.P. Chi, S.K. Choi and J.S. Park, 'Structurally stable flows', Bull. Austral. Math. Soc. 45 (1992), 79-90.

[3] C. Conley, Isolated invariant sets and the Morse index, C.B.M.S. publication 38 (American Mathematical Society, Providence, RI, 1978).

[4] M. Hurley, 'Consequences of topological stability', J. Differential Equations 54 (1984), 60-72.

[5] M. Hurley, 'Combined structural and topological stability are equivalent to Axiom A and the strong transversality condition', Ergodic Theory Dynamical Systems 4 (1984), 81-88.

[6] M. Hurley, 'Fixed points of topologically stable flows', Trans. Amer. Math. Soc. 294 (1986), 625-633.

[7] S. Liao, 'Obstruction sets (II)', Acta Sci. Natur. Univ. Pekinensis 2 (1981), 1-36.

[8] R. Mañé, 'An ergodic closing lemma', Ann. of Math. 116 (1982), 503-540.

[9] R. Mañé, 'A proof of the $C^{1}$ stability conjecture', Publ. I.H.E.S. 66 (1987), 161-210.

[10] L. Markus, Lectures in differentiable dynamics, C.B.M.S. publication 3 (American Mathematical Society, Providence, RI, 1980).

[11] J. Palis Jr. and W. de Melo, Geometric theory of dynamical systems (Springer-Verlag, Berlin, Heidelberg, New York, 1982).

[12] L. Wen, 'Combined two stabilities imply Axiom A for vector fields', Bull. Austral. Math. Soc. 48 (1993), 23-30.

Department of Mathematics

Case Western Reserve University

Cleveland OH 44106-7058

United States of America 